\title{
New Technique for the Measurement of Magnetic Critical Exponent $\beta^{*}$
}

\author{
Ping Sheng \\ Institute for Advanced Study, Princeton, New Jersey 08540
}

and

C. N. Manikopoulos $\dagger$ and T. R. Carver

Joseph Henry Laboratories of Physics, Princeton University, Princeton, New Jersey 08540

(Received 30 November 1972)

\begin{abstract}
The critical exponent $\beta$ of Gd is measured directly for the first time by a new technique of ferromagnetic transmission resonance in zero external field. The technique consists of measuring the microwave power transmitted through a thin Gd film $(47 \mu \mathrm{m})$ as a function of temperature. Analysis of the resulting line shape yields conductivity $\sigma=0.745$ $\times 10^{16} \mathrm{sec}^{-1}$, spin relaxation time $\tau=7.3 \times 10^{-11} \mathrm{sec}$, Curie temperature $T_{\mathrm{C}}=295^{\circ} \mathrm{K}$, and critical exponent $\beta=0.31 \pm 0.05$.
\end{abstract}

In this Letter we describe a measurement of the critical exponent $\beta$ for gadolinium defined by $M(T) / M(0)=B\left(1-T / T_{\mathrm{C}}\right)^{\beta}$ for $T \rightarrow T_{\mathrm{C}}$. This measurement is distinguished by being carried out by means of a new technique, possibly applicable to other ferromagnets like iron and nickel, and by the fact that it is the first direct determination of this exponent for gadolinium. The measurement is less distinguished in accuracy, however, since as yet it does not approach the 1-5\% accuracy of the nuclear resonance technique in antiferromagnets and Mössbauer-effect measurements in a few substances.

Our new technique involves the observation of a zero-field resonance in a thin single crystal of gadolinium by means of the microwave resonance transmission technique which was developed earlier to study conduction-electron spin resonance and skin-depth modulation in metals., ${ }^{1,2}$ Since it does involve a zero $H$ field, one must sweep through the resonance by varying the temperature instead of the field and this determines $M(T)$ through interpretation of the resulting line shape. In this Letter we will give an outline of the theoretical considerations and the experimental method for the new technique. A more detailed account will be published elsewhere. ${ }^{3}$

Consider a ferromagnetic single crystal in the form of a film of thickness $l$, with its surface lying in the $x z$ plane. The easy axis of magnetization $M$ is taken to be along the $z$ axis, and the applied microwaves are assumed to be plane waves normally incident upon the $x z$ plane, the tangential component of the magnetic vector being along the $x$ axis. In this work we will be concerned only with the transmission of electromagnetic waves through the sample. The effects of exchange interaction, anisotropy energy, and magnetostriction are analyzed elsewhere ${ }^{3}$ and can be shown not to affect the main results of the present work.

Calculation of the ratio of the transmitted magnetic vector to the incident magnetic vector, $h_{3} / h_{1}$, proceeds in two steps: (i) Calculate the wave vector $k_{2}$ inside the sample as a function of frequency $\omega$, and (ii) fit boundary conditions to obtain the desired expression for $h_{3} / h_{1}$. One needs only Maxwell's equations and Bloch's phenomenological equation. Eliminating the electric field from Maxwell's equations, we have

$$
\begin{aligned}
& \nabla(\nabla \cdot \overrightarrow{\mathrm{H}})-\nabla^{2} \overrightarrow{\mathrm{H}}=-\left(4 \pi \sigma / c^{2}\right) \partial(\overrightarrow{\mathrm{H}}+4 \pi \overrightarrow{\mathrm{M}}) / \partial t, \\
& \partial \overrightarrow{\mathrm{M}} / \partial t=\gamma \overrightarrow{\mathrm{M}} \times \overrightarrow{\mathrm{H}}-\overrightarrow{\mathrm{M}} \\
& \text { tr ansver se }
\end{aligned}
$$

where the displacement current has been neglected because of the large value of $\sigma$.

Here $\gamma\left(=1.72 \times 10^{7} \mathrm{~Hz} / \mathrm{G} \text { for } \mathrm{Gd}\right)^{4}$ is the gyromagnetic ratio, $c$ the speed of light, $\sigma$ the conductivity, $\tau$ the phenomenological spin relaxation time (typically $10^{-10} \mathrm{sec}$ ), ${ }^{5}$ and $\overrightarrow{\mathrm{M}}$ and $\overrightarrow{\mathrm{H}}$ are the magnetization and magnetic field vectors, respectively. If we write $\overrightarrow{\mathrm{M}}=m_{x} \hat{i}+m_{y} \hat{j}+M \hat{k}$ and $\overrightarrow{\mathrm{H}}=h_{x} \hat{i}+h_{y} \hat{j}$, where the components $m_{x}, m_{y}, h_{x}$, and $h_{y}$ are understood to be small and proportional to $\exp \left[i\left(k_{2} y-\omega t\right)\right]$, a nontrivial solution of Eqs. (1) and (2) is obtained if

$$
k_{2}{ }^{2}=\frac{2 i}{\delta^{2}}=\left[1+\frac{R^{2}}{(\lambda-i \Omega)^{2}}\right],
$$

where $\delta^{2}=c^{2} / 2 \pi \sigma \omega, R=M(T) / M(0), \lambda=[1 / 4 \pi M(0)]$ $\times 1 / \gamma \tau$, and $\Omega=\omega / 4 \pi M(0) \gamma$.

One should note that for frequencies much higher than $4 \pi M(0) \gamma$ the solution (3) becomes identical with the usual skin-depth solution $k_{2}=(1+i) /$ 
$\delta$; and for slow spin relaxation $(\lambda \ll 1), k_{2}$ goes to zero as $\omega$ approaches $4 \pi M(T) \gamma$. A physical picture of the interactions between the electromagnectic field and the spin system is as follows.

If $\lambda \ll 1$, we see that at $\omega \simeq 4 \pi M(T) \gamma, R=\Omega$, so that from Eqs. (1) and (2), $h_{y}=-4 \pi m_{y}$ and $h_{x}$ $\simeq-4 \pi m_{x}$. In other words, at $\omega \simeq 4 \pi M(T) \gamma, b_{x, y}$ $\simeq 0$ and $-\overrightarrow{\mathrm{m}} \cdot \overrightarrow{\mathrm{h}}$, the energy of the spin system, is at a maximum. In order to understand the effect of $b_{x, y} \simeq 0$ on the energy transfer, we proceed to fit the boundary conditions (the parallel components of $h$ and $e$ are continuous across the sample boundary). A simple calculation yields

$$
\frac{h_{3}}{h_{1}}=-i \frac{2 \omega \delta^{2}}{c} \frac{k_{2}}{\left(f_{-}-f_{+}\right)},
$$

where

$$
f_{ \pm}=\left[1 \pm i\left(k_{2} \delta^{2} \omega / 2 c\right)\right]^{2} \exp \left( \pm i k_{2} l\right) .
$$

The quantity experimentally observed is $\left|h_{3}\right|$ $h_{1} \mid,{ }^{1,3,5}$ the magnitude of $h_{3} / h_{1}$. It should be noted that the expression for $\left|h_{3} / h_{1}\right|$ has a maximum at $\omega \simeq 4 \pi M(T) \gamma$. Near the maximum the electric and magnetic fields have the following configurations inside the sample if $\lambda=0: e_{z} \simeq(c / 4 \pi \sigma)\left(2 h_{1 x} /\right.$ $l$ ) and $h_{x} \simeq 2 h_{1 x}\left(1-y / l^{\prime}\right)$, where $h_{1 x}$ is the $x$ component of the incident magnetic vector, $l^{\prime}=l+\omega \delta^{2} /$ $2 c$, and $y$ is the distance from the incident surface. The energy flow, which is proportional to $\overrightarrow{\mathrm{e}} \times \overrightarrow{\mathrm{h}}$, decreases linearly as a function of distance inside the sample. The physics is therefore very clear: Because part of the energy is stored in the spin system, only that part of the energy contained in the electromagnetic fields will be dissipated (since energy dissipation is proportional to $\overrightarrow{\mathrm{E}}^{2}$ ). This decreased attenuation gives rise to an enhanced transmission. In this context the frequency $\omega=4 \pi M(T) \gamma$ is physically that frequency at which the energy of the spin system is maximum.

The microwave transmission apparatus used in this work is identical to the one used to observe conduction electron spin resonance and has already been discussed in detail elsewhere. ${ }^{1,5}$ The apparatus consists of two microwave cavities, one the exciting cavity and the other the receiving cavity, with the sample forming the common wall between them. A superheterodyne receiver is attached to the receiving cavity. The only addition to the apparatus in the present work is a continuously variable motor-driven $360^{\circ}$ phase shifter at the output of the local oscillator which allows us to measure the transmitted microwave signal power rather than the phase-dependent amplitude conventionally detected. The sample we used was cut from an extremely pure single-crystal gadolinium button of size $\frac{1}{4} \times \frac{1}{4} \times \frac{1}{8}$ in. ${ }^{3}$ generously provided to us by Dr. F. H. Spedding. The crystal was cut into thin slices with a diamond disk saw and then chemically lapped to foils of thickness between 25 and $50 \mu \mathrm{m}$. The thickness of each foil was established by measuring its surface area and its weight. The sample was subsequently mounted on a goniometer and oriented by $x$-ray crystallography techniques. The $c$ axis of the crystal sample was found to be inclined to the surface at an angle $\alpha=15^{\circ}$ with accuracy better than $1^{\circ}$. The temperature of the sample was controlled with a conventional Wheatstone bridge circuit with a $10-\mathrm{k} \Omega$ thermistor mounted on the cavity block as the control element, a $10-k \Omega \mathrm{He}-$ lipot as the adjustable resistor set to the corresponding temperature desired, and two $10-\mathrm{k} \Omega$ precision resistors as reference standards. When the cavity block temperature is lower than the desired temperature, the bridge unbalances and it turns on a zero-voltage crossing switch which provides a gate pulse to trigger a Triac. The gate turns off when the cavity temperature becames higher than the set temperature. The Triac feeds about $5 \mathrm{~W}$ to the three resistors mounted on cavity block. We can thus suppress temperature drifts of the cavity system to less than 50 mdeg as determined by monitoring an iron-Constantan thermocouple soldered on the cavity block. Two more iron-Constantan thermocouples measure the temperatures of the exciting and receiving cavity, respectively. The temperature of the sample was taken as the mean of the two thermocouple readings. We observed that the exciting cavity is hotter than the receiving cavity by as much as $1 \mathrm{deg}$ as a result of the disssipation of $300 \mathrm{~mW}$ incident $\mathrm{rf}$ power in the exciting cavity. However, calculation ${ }^{3}$ shows that the actual power dissipated at the sample is less than $1 \mathrm{~mW}$. This implies that the temperature gradient across the sample is considerably less than $40 \mathrm{mdeg}$, well within our temperature control error. Therefore, the absolute sample temperature measurements may suffer by an error of $1 \mathrm{deg}$. However, the relative temperature drifts, to which the extraction of $\beta$ is sensitive, were observed to be no more than $50 \mathrm{mdeg}$.

We note that in Fig. 1 the data on $\left|h_{3} / h_{1}\right|$ agree in general shape with those calculated from the theory. The line shape as a function of temperature consists of three parts. Above the Curie 


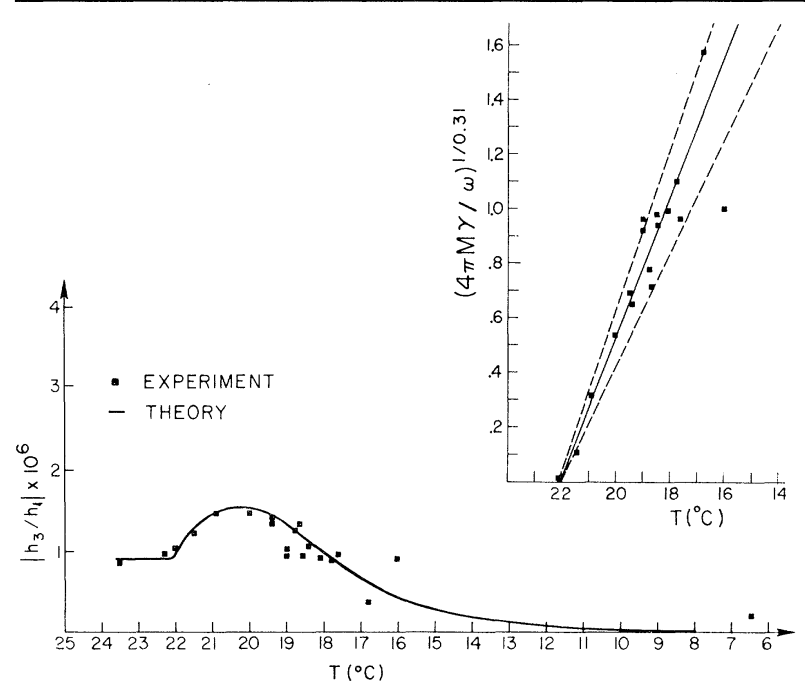

FIG. 1. $\left|h_{3} / h_{1}\right|$ plotted as a function of temperature $T$. The inset shows $[4 \pi M(T) \gamma / \omega]^{-1 / 0}, 31$ plotted as a function of $T . M(T)$ is obtained from $\left|h_{3} / h_{1}\right|$ through Eq. (4). Dashed lines indicate the slopes from which the credible limits of $B$ are obtained.

temperature the transmitted power is a slowly varying function of temperature. We call this region I. As temperature is lowered through $\boldsymbol{T}_{C}$, the transmitted power increases within 1 or 2 degrees (region II) to a peak and then slowly decreases as the temperature is further reduced (region III). In order to fit the data, we will proceed in three steps. The first step is to note that in Eq. (3) when $M=0, k_{2}$ reduces to $(1+i) / \delta$, which is the usual skin-depth attenuation. Therefore, from the data in region I, thickness $l=47$ $\mu \mathrm{m}$, and $\omega=5.74 \times 10^{10} \mathrm{rad} / \mathrm{sec}$, we obtain $\sigma$ $=0.745 \times 10^{16} \mathrm{sec}^{-1}$ (corresponding to a value of resistivity $\left.\rho=121 \times 10^{-6} \Omega \mathrm{cm}\right)$. This value of $\sigma$ compares satisfactorily with the result of Nigh, Legvold, and Spedding, ${ }^{6}$ in which the resistivity is $119 \times 10^{-6} \Omega \mathrm{cm}$ along the $c$ axis. In the second step, we note that the ratio of transmitted signal at peak to the signal at region I depends only on the value of relaxation time $\tau$. From the data we obtain $\tau=7.25 \times 10^{-11} \mathrm{sec}$. With the above values of $\sigma$ and $\tau$, each datum point can now be associated with a unique value of $M$ through Eq. (4). The values for $B$ and $\beta$ are then obtained by plotting $\ln M$ versus $\ln \left(T_{C}-T\right)$ and $M^{1 / 3}$ as a function of $T$ (see the insert to Fig. 1) for various values of $T_{C}$ until the best fit to the data gives straight lines on both plots. The above fitting procedure yields $M(T) / M(0)=0.49\left(1-T / T_{C}\right)^{0.31}$ and $T_{C}=295^{\circ} \mathrm{K}$. The value $\beta=0.31$ is the mean of the credible limits indicated by dashed lines in Fig. $2-\beta=0.36$ and
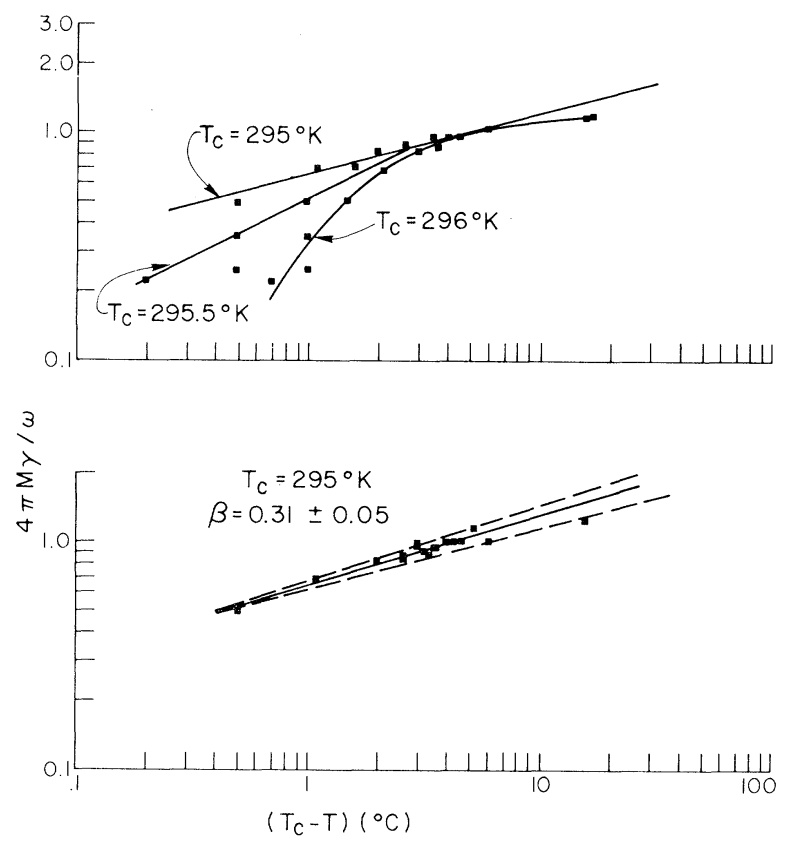

FIG. 2. $4 \pi M(T) \gamma / \omega$ plotted as a function of $\left(T_{C}-T\right)$. The upper figure shows the effect of choosing different values of $T_{C}$. The lower figure shows the best fit to the data. Dashed lines indicate the credible limits of the value $\beta$. Because of crowding, not all the data points are displayed in the upper figure.

$\beta=0.26$. The value $B=0.49$ is the mean of $B=0.52$ and $B=0.46$ (indicated by dashed lines in the inset to Fig. 1), where we have used the value of $M(0)$ $=2010 \mathrm{G} .{ }^{7}$ Plotting the data with different $T_{\mathrm{C}}$ and $\beta$ indicates that the above limits are generously adequate.

When the sample consists of several domains with the directions of magnetization reversed from one domain to the next it might be expected that there is some loss when signals cross the Bloch walls. This loss can be viewed as a shortening of the relaxation time $\tau$, which simply distributes the loss uniformly across the sample. Similarly, the tilting of the $c$ axis out of the sample surface means that the transmission mode, instead of propagating into the sample at $90^{\circ}$ with respect to the sample surface, now propagates at $90^{\circ}-15^{\circ}$ (where $15^{\circ}$ is the tilting angle) with respect to the sample surface. The net effect is a loss of signal strength which can again be described by a shortening of $\tau$. We therefore expect the value $\tau \simeq 7.3 \times 10^{-11} \mathrm{sec}$ to be shorter than the true value.

We wish to thank Dr. F. H. Spedding for providing the sample material and Dr. Paul Schmidt for suggesting the chemical lapping technique. 
One of us (P.S.) wishes to acknowledge helpful discussions with Dr. T. C. McGill and to thank Dr. Carl Kaysen for the hospitality at the Institute for Advanced Study.

*Work supported by the National Science Foundation under Grants No. GP-16147 A\# 1 and No. GP-19891.

$\dagger$ Present address: Department of Physics, State University of New York at Buffalo, Buffalo, N. Y. 14214.

${ }^{1}$ R. B. Lewis and T. R. Carver, Phys. Rev. 155, 309 (1967).
${ }^{2}$ G. C. Alexandrakis, T. R. Carver, and O. Horan, Phys. Rev. B 5, 3472 (1972).

${ }^{3}$ C. N. Manikopoulos, P. Sheng, and T. R. Carver, to be published.

${ }^{4}$ A. F. Kip, C. Kittel, A. M. Portis, R. Barton, and F. H. Spedding, Phys. Rev. $\underline{89}, 518$ (1953).

${ }^{5} \mathrm{G}$. C. Alexandrakis, thesis, Princeton University, 1968 (unpublished) .

${ }^{6}$ H. E. Nigh, S. Legvold, and F. H. Spedding, Phys. Rev. 132, 1092 (1963).

${ }^{7} \mathrm{C}$. Kittel, Introduction to Solid State Physics (Wiley, New York, 1966), p. 461.

\title{
Exact Multisoliton Solutions of the Self-Induced Transparency and Sine-Gordon Equations
}

\author{
P. J. Caudrey, J. D. Gibbon, J. C. Eilbeck, and R. K. Bullough \\ Department of Mathematics, University of Manchester Institute of Science and Technology, \\ Manchester, M60 1QD, Great Britain \\ (Received 11 December 1972)
}

We confirm that the previously conjectured $N$-soliton solution of the self-inducedtransparency equations is a solution for all $N$. The solution is transformed to give an exact solution of the sine-Gordon equation describing multiple collision of $N$ kinks with different velocities. Simple asymptotic forms of both solutions are given.

In a recent paper, ${ }^{1}$ an $N$-soliton solution was proposed for the self-induced-transparency (SIT) equations. The SIT equations can be reduced on resonance and in the sharp line limit to the dimensionless form ${ }^{2,3}$

$$
\begin{aligned}
& E_{x}+E_{t}=\alpha P, \\
& P_{t}=E N, \\
& N_{t}=-E P .
\end{aligned}
$$

The notation is that of Ref. 1 . The boundary conditions are $E(x, t), P(x, t) \rightarrow 0 ; N(x, t) \rightarrow-1$ (attenuator) as $x \rightarrow \pm \infty . P^{2}+N^{2}$, the trace of the atomic density matrix, is a constant of integration and equal to unity.

A proof that the solution given in Ref. 1 is exact for all $N$ has now been found; full details will be published elsewhere. ${ }^{4}$ The amplitudes $E_{i}$ of the $N$ solitons are restricted such that $E_{i} \neq 0$, $E_{i} \neq \pm E_{j}$. A full treatment of the applications of this solution in nonlinear optics will be published elsewhere.

The $N$-soliton solution of the SIT equations has more general applications since it gives a solution of the sine-Gordon (SG) equation. The SG equation can be derived from the SIT equations by the following simple transformation ${ }^{3}$ :

$$
\begin{aligned}
& x^{\prime}=\alpha^{1 / 2}(t-2 x), \quad t^{\prime}=\alpha^{1 / 2} t \\
& E=\partial \sigma / \partial t, \quad P=-\sin \sigma, \quad N=-\cos \sigma .
\end{aligned}
$$

Dropping the primes, (1) becomes, using (2) and (3),

$$
\sigma_{x x}-\sigma_{t t}=\sin \sigma .
$$

Equation (4) is the sine-Gordon equation which occurs in many branches of physics. ${ }^{5-7}$ The SIT solution transforms into an exact solution of the SG equation in the transformed $x, t$ coordinates:

$$
\begin{aligned}
& \sigma(x, t)=\arccos \left[1+2\left(\frac{\partial^{2}}{\partial t^{2}}-\frac{\partial^{2}}{\partial x^{2}}\right) \ln f(x, t)\right], \\
& f(x, t)=\operatorname{det}|M(x, t)| .
\end{aligned}
$$

The $N \times N$ matrix $M_{i j}$ has elements

$$
M_{i j}=\frac{\left(a_{i} a_{j}\right)^{1 / 2}}{a_{i}+a_{j}}\left[\exp \left(\theta_{i}\right)+(-1)^{i+j} \exp \left(-\theta_{j}\right)\right]
$$

where

$$
\theta_{i}=\frac{1}{2}\left[\left(a_{i}-\frac{1}{a_{i}}\right) t+\left(a_{i}+\frac{1}{a_{i}}\right) x\right]+\delta_{i}
$$

and the $a_{i}$ and $\delta_{i}$ are arbitrary constants. 